\title{
Estudo de validação sobre dificuldade visual autorrelatada entre estudantes dos cursos de graduação da Universidade Federal de Pelotas
}

\author{
Validation study on self-reported visual impairment among \\ undergraduate students at the Federal University of Pelotas
}

Juliana das Chagas Meroni Tissot (https://orcid.org/0000-0001-9094-9049) ${ }^{1}$ Bruna Gonçalves Cordeiro da Silva (https://orcid.org/0000-0003-2917-7320) ${ }^{1}$ Ana Maria Baptista Menezes (https://orcid.org/0000-0002-4129-0898) ${ }^{1}$
${ }^{1}$ Programa de PósGraduação em Epidemiologia, Universidade Federal de Pelotas. R. Prof. Dr. Araújo 538, Centro. 96020360 Pelotas RS Brasil. julianameroni@gmail.com

\begin{abstract}
The objectives of the study were to assess the prevalence of self-reported visual impairment among undergraduate students between 18 and 39 years of age at the Federal University of Pelotas, and to validate a question about visual impairment in a subsample of the participants. A cross-sectional study was conducted by asking the question "Do you have any difficulty seeing up close and/or in the distance?" As the gold standard for the validation study, visual acuity (VA) was measured using the Snellen chart. Patients with $V A$ less than 20/40 in either eye were considered to have reduced VA. The prevalence of self-reported visual impairment was $37.3 \%$ (95\% CI: 35.1 39.6) and VA less than 20/40 in either eye was $6.9 \%$ (95\% CI: 5.3-8.9). The question revealed sensitivity of $71.4 \%$ (95 CI: 57.8-82.7), specificity of $66.9 \%$ (95\% CI: 63.4-70.2), positive predictive value of $13.8 \%$ (95\% CI: 10.0-18.3), and negative predictive value of 96.9\% (95\% CI: 95,1-98.2). The results indicated a high prevalence of self-reported visual impairment among university students. The question showed reasonable sensitivity and specificity and high negative predictive value and may be used for screening for ophthalmological evaluation among young adult university students.
\end{abstract}

Key words Sensitivity and specificity, Vision disorders, Surveys and questionnaires, Visual acuity
Resumo Os objetivos do estudo foram avaliar a prevalência de dificuldade visual autorrelatada entre acadêmicos de 18 a 39 anos de idade da Universidade Federal de Pelotas e realizar a validação de uma pergunta sobre dificuldade visual em uma subamostra dos participantes, com dados coletados entre novembro de 2017 e julho de 2018. Realizou-se um estudo transversal com a pergunta "Você tem alguma dificuldade para enxergar de perto elou de longe?". Como padrão-ouro para o estudo de validação, a acuidade visual (AV) foi medida através da tabela de Snellen. Considera-se AV alterada quando menor que 20/40 em qualquer olho. A prevalência de dificuldade visual autorrelatada foi de 37,3\% (IC95\%: 35,1-39,6) e a de AV menor que 20/40 em qualquer olho foi 6,9\% (IC95\%: 5,3-8,9). A pergunta apresentou sensibilidade 71,4\% (IC95\%: 57,8-82,7), especificidade 66,9\% (IC95\%: 63,4-70,2), valor preditivo positivo 13,8\% (IC95\%: 10,0-18,3) e valor preditivo negativo 96,9\% (IC95\%: 95,1-98,2). Os resultados indicaram alta prevalência de dificuldade visual autorrelatada entre os universitários e pergunta com sensibilidade e especificidade razoáveis e com alto valor preditivo negativo, podendo ser utilizada como triagem para consulta com oftalmologista e em estudos epidemiológicos com jovens adultos universitários.

Palavras-chave Sensibilidade e especificidade, Transtornos da visão, Inquéritos e questionários, Acuidade visual 


\section{Introdução}

A acuidade visual (AV) pode ser definida como a quantificação da capacidade de discriminação de formas e contrastes, sendo um dos parâmetros que melhor representa o desempenho da função visual. A percepção visual é a decodificação dos sinais recebidos e a transformação desses em imagem, e que depende da chegada do estímulo visual ao córtex e da cognição, ou seja, do entendimento do significado desta imagem. Alterações em quaisquer partes do sistema visual irão alterar a $\mathrm{AV}$, que poderá também ser afetada por estados emocionais, mentais e educacionais ${ }^{1}$.

Estimativas de deficiência visual baseadas na população mundial em 2015 apontam prevalência de $5,5 \%$ de deficiência visual ${ }^{2}$, e que a principal causa desta deficiência em indivíduos com 50 anos de idade ou menos são erros refrativos não corrigidos ${ }^{3}$. A Pesquisa Nacional de Saúde de 2013, um inquérito brasileiro de base populacional, relatou prevalência de deficiência visual autorreferida de 5,9\% na região Sul do país e 5,2\% em indivíduos com menos de 40 anos de idade. Um estudo realizado entre universitários de Gana mostrou prevalência de 3\% de deficiência visual bilateral, sendo que $96 \%$ destes era devido a erro refrativo não corrigido ${ }^{5}$. Não foram encontrados estudos brasileiros sobre o tema.

A presença de deficiência visual tem impacto negativo em diversos aspectos da vida dos indivíduos. Estudos brasileiros mostraram a associação de baixa AV com baixo rendimento escolar em crianças ${ }^{6-9}$. Além disso, estudos internacionais mostraram que esta condição também esteve associada a transtornos de ansiedade ${ }^{10}$ e ideação suicida ${ }^{11}$. Sabendo-se que a maior causa de deficiência visual é erro refrativo, uma condição reversível com óculos ou lente de contato, a deteç̧ão e tratamento adequado destes indivíduos pode trazer melhora na sua qualidade de vida.

A Sociedade Brasileira de Oftalmologia recomenda um exame na infância, um na idade adulta entre 30 e 40 anos e exames anuais a partir dos 40 anos $^{12}$. De acordo com a Sociedade Canadense de Oftalmologia, para indivíduos entre 19 e 40 anos de baixo risco para doenças oculares e assintomáticos, a indicação de triagem oftalmológica é a cada 10 anos $^{13}$. De forma semelhante, a Academia Americana de Oftalmologia recomenda que, antes dos 40 anos de idade, a consulta oftalmológica deva ser realizada a cada 5 a 10 anos, ou menos, se apresentarem algum sintoma ou fator de risco conhecido para doença ocular ${ }^{14}$. Exames anuais após os 40 anos são também indicados pe- las entidades canadense e americana. Não foram encontradas recomendações formais para a faixa dos 18 aos 30 anos de idades no Brasil.

Assim, adultos jovens não são prioridade para exames de rotina, a não ser que estes façam parte de algum grupo de risco ou que percebam alguma alteração em sua função ocular ${ }^{14}$. Com base nisto e nas implicações da deficiência visual anteriormente mencionadas, a identificação dos casos de deficiência visual através de uma pergunta simples, no lugar de exames de AV, que necessitam treinamento de equipe, maior tempo e maior custo relativo, pode ser de grande utilidade, e pode facilitar a detecção de possíveis deficientes visuais e o seu respectivo encaminhamento para serviços oftalmológicos.

Diversos instrumentos já foram desenvolvidos para avaliar a função visual, como o National Eye Institute Visual Function Questionnaire (NEI-VFQ-25) ${ }^{15}$, com 25 questões e o Activities of Daily Vision Scale (ADVS) ${ }^{16}$, adaptado para o português por Simão e colaboradores ${ }^{17}$, com questões sobre 21 atividades diárias. Instrumentos menores, com apenas uma questão, também já foram desenvolvidos a fim de avaliar alterações de AV. Em Pelotas, Rio Grande do Sul, Duarte e colaboradores $^{18}$ elaboraram uma pergunta para rastreio de dificuldade visual para perto entre adultos com 30 anos ou mais, e obteve-se sensibilidade e especificidade de $86 \%$ e $72,6 \%$, respectivamente ${ }^{18}$. Para fins de validação da referida pergunta, foi utilizado como padrão-ouro a tabela de Jaeger. Recentemente, no Japão, foi desenvolvido o paper-based visual acuity (PBVA), um instrumento composto por apenas uma pergunta e considerado pelos autores útil na triagem de indivíduos com baixa AV e, também, para estudos populacionais ${ }^{19}$. Contudo, estes instrumentos foram validados em populações predominantemente mais velhas, com idades em que o exame oftalmológico de rotina já tem indicação.

O presente estudo foi realizado como parte do consórcio de pesquisa Saúde dos Estudantes Universitários da Universidade Federal de Pelotas (SEU -UFPel), um estudo observacional transversal. Os objetivos desse estudo foram avaliar a prevalência de dificuldade visual autorrelatada entre acadêmicos da UFPel ingressantes no primeiro sementre de 2017, e realizar um estudo de validação de uma pergunta sobre dificuldade visual, através da aplicação da tabela de Snellen, em uma subamostra do consórcio, a fim de estabelecer a sensibilidade, a especificidade e os valores preditivos positivo e negativo para essa pergunta, para fins de uso em estudos epidemiológicos e como auxiliar no ras- 
Foram elegíveis para o estudo todos os alunos ingressantes no primeiro semestre letivo de 2017 dos cursos de graduação presenciais da UFPel, matriculados no segundo semestre de seu respectivo curso e com 18 anos de idade completos ou mais. A coleta dos dados foi realizada entre novembro de 2017 e julho de 2018.

Para o cálculo do tamanho de amostra do estudo de validação, utilizou-se uma prevalência do desfecho na população de $10 \%$, intervalo de confiança de $95 \%$ com amplitude de 10 pontos percentuais e valores esperados de sensibilidade e especificidade ${ }^{20}$ de $80 \%$, obtendo-se um número final de 615 indivíduos. Para o estudo de prevalência de dificuldade visual autorrelatada, foram considerados prevalência de 15\%, significância de $5 \%$ e erro de 2 pontos percentuais, obtendo-se 938 indivíduos.

Utilizou-se um questionário geral anônimo, autoaplicado, com variáveis demográficas e socioeconômicas, além de questões sobre os diversos temas do consórcio. Com relação à dificuldade visual autorrelatada, havia uma pergunta filtro: Você usa algum tipo de lente/óculos para enxergar melhor?. Para aqueles que responderam não, a pergunta sobre dificuldade visual era: Você tem alguma dificuldade para enxergar de perto el ou de longe? ${ }^{18}$; e, para aqueles que utilizavam alguma correção óptica (óculos ou lentes de contato), a pergunta era: Usando seus óculos ou lentes de contato, você tem alguma dificuldade para enxergar de perto e/ou de longe? com as seguintes opções de resposta: não, sim de perto, sim de longe ou sim ambos. Logo após, um a cada dois participantes foi encaminhado ao teste de AV para o estudo de validação, independente da resposta à pergunta sobre dificuldade visual. Para fins de análise, foram considerados positivos para dificuldade visual autorrelatada todos que responderam sim de perto, sim de longe ou sim ambos.

Como padrão-ouro para o teste da $\mathrm{AV}$, foi utilizada a tabela de Snellen posicionada a 6 metros de distância do participante, em ambiente iluminado. Foi considerado como resultado do teste de AV a linha contendo as letras (optotipos) de menor tamanho na qual o indivíduo conseguiu identificar corretamente pelo menos metade delas $^{21}$. A determinação da acuidade foi realizada com os óculos vigentes ou lentes de contato, naqueles que o utilizavam, chamada acuidade visual presente. Para categorização da $\mathrm{AV}$, foram adotados critérios baseados no recomendado pela $\mathrm{OMS}^{22}$. Os critérios utilizados no teste para considerar o participante como portador de $\mathrm{AV}$ diminuída foram: AV menor que 20/40, 20/70 ou 20/200, em qualquer olho.

Indivíduos com 40 anos de idade ou mais foram excluídos das análises e, posteriormente, aqueles usuários de correção óptica que não estavam com seus óculos ou lentes de contato no momento da realização do teste de AV foram excluídos do estudo de validação. O teste de acuidade visual foi conduzido por uma aplicadora treinada. Foi realizado controle de qualidade dos testes de $\mathrm{AV}$, por oftalmologista, em $8,9 \%$ dos participantes.

Para caracterização da amostra geral do consórcio e do estudo de validação, foram utilizadas frequências brutas e relativas para as variáveis de idade, coletadas em anos completos e categorizada em três grupos para análise: entre 18 e 19, 20 e 22, 23 e 39 anos, sexo, cor da pele autorrelatada (branca, preta, parda ou outras), estado civil (solteiro, casado ou em união estável, separado ou divorciado, ou viúvo), classe social de acordo com a classificação da Associação Brasileira de Empresas de Pesquisa (ABEP), e quanto ao uso ou não uso de correção óptica (óculos ou lentes de contato).

Foram avaliados sensibilidade, especificidade, valores preditivos positivo e negativo e seus respectivos intervalos de confiança de 95\%. A análise de concordância, para controle de qualidade do teste padrão-ouro, foi calculada através da estatística kappa. As prevalências da dificuldade visual autorrelatada e AV menor que 20/40 em qualquer olho e seus intervalos de confiança de 95\% foram calculadas para cada categoria das variáveis idade, sexo, cor da pele, estado civil, classe social e uso de correção óptica, e foi realizado teste qui-quadrado para avaliar diferenças nas prevalências entre as categorias dessas variáveis. Foi utilizado o software Stata versão 15.1 (StataCorp, College Station, TX, EUA) para todas as análises.

O presente estudo foi aprovado pelo Comitê de Ética da Faculdade de Medicina da UFPel, e todos participantes assinaram um Termo de Consentimento Livre e Esclarecido. Além disso, todos os participantes que apresentaram $\mathrm{AV}$ menor que 20/30 foram orientados a buscar atendimento oftalmológico por possível alteração visual. 


\section{Resultados}

A taxa de resposta ao consórcio universitário foi de $69 \%$ e, dentre os respondentes selecionados para o teste de $\mathrm{AV}$, a taxa de resposta foi de $95,9 \%$. As perdas e recusas do consórcio foram maiores entre indivíduos com 23 anos ou mais $(46,7 \%)$ e do sexo masculino (52,8\%). A Figura 1 mostra o fluxograma dos participantes durante o estudo.
Na Tabela 1 estão descritas as características da amostra geral do consórcio, além das prevalências de dificuldade visual autorrelatada entre estes e de AV menor que 20/40 em qualquer olho entre os participantes do estudo de validação. Entre os 1.769 participantes do consórcio, a maioria era do sexo feminino $(54,9 \%)$, solteira $(93,1 \%)$, de cor da pele branca $(71,9 \%)$, pertencente à classe social B $(43,7 \%)$ e não usuária de

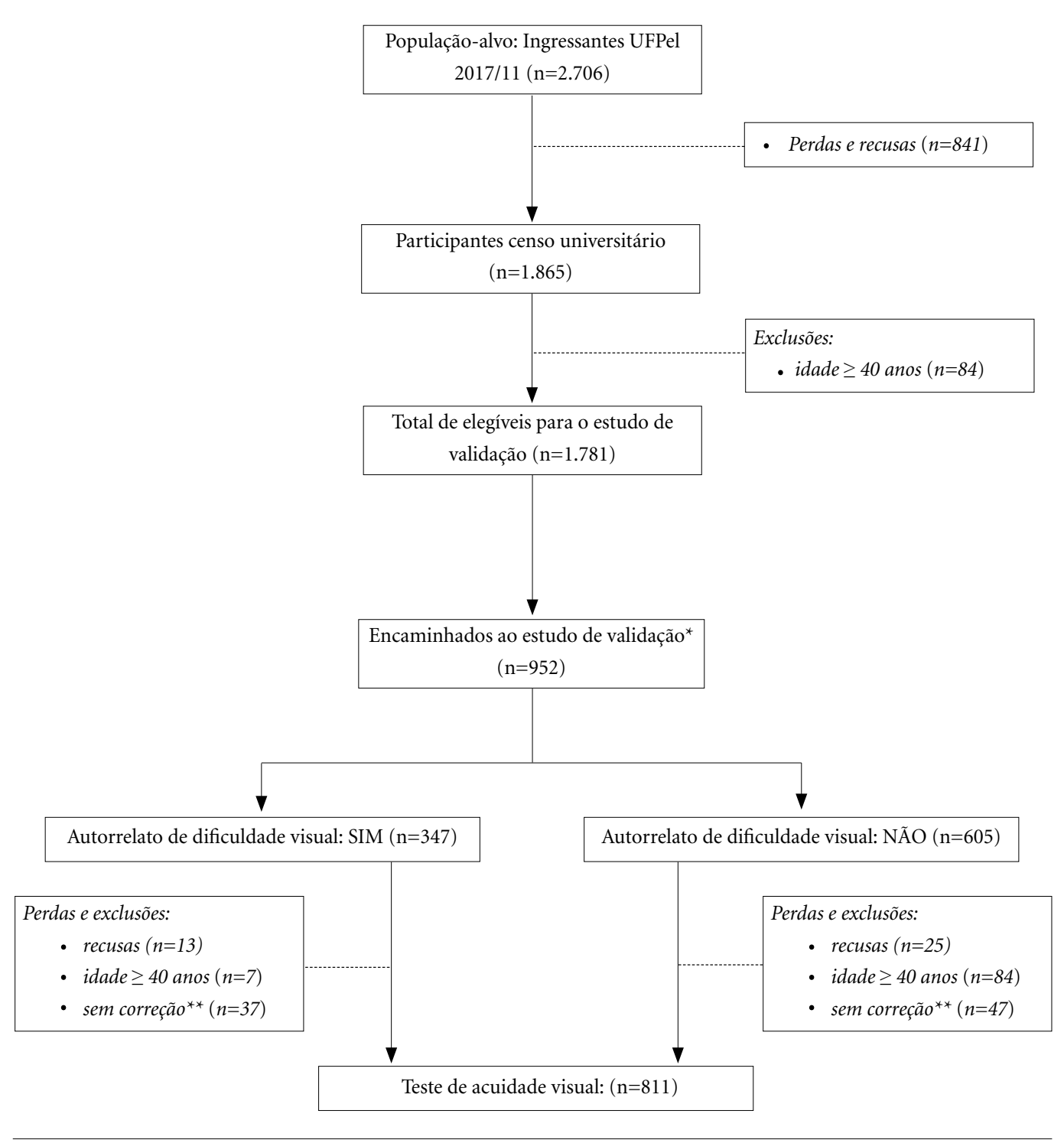

Figura 1. Fluxograma do estudo de validação sobre acuidade visual na amostra de estudantes da Universidade Federal de Pelotas/RS.

\footnotetext{
${ }^{1}$ População-alvo do censo: ingressantes na Universidade Federal de Pelotas no primeiro semestre letivo de 2017 e que estivessem matriculados na universidade no segundo semestre letivo do mesmo ano. ${ }^{\star}$ Excluídos 4 participantes que não responderam à pergunta a ser validada. ${ }^{\star *} 84$ excluídos por usarem óculos e não estarem com óculos no momento do teste.
} 
Tabela 1. Características e prevalências de dificuldade visual autorrelatada e de acuidade visual menor a 20/40 em um ou em ambos olhos, nas amostras dos estudantes da Universidade Federal de Pelotas, Rio Grande do Sul.

\begin{tabular}{|c|c|c|c|c|}
\hline \multirow{3}{*}{ Características } & \multicolumn{2}{|c|}{$\begin{array}{l}\text { Amostra estudo de prevalência } \\
\qquad(\mathrm{N}=1.769)\end{array}$} & \multicolumn{2}{|c|}{$\begin{array}{l}\text { Amostra estudo de validação }{ }^{1} \\
\qquad(\mathrm{~N}=811)\end{array}$} \\
\hline & N (\%) & $\begin{array}{l}\text { Dificuldade visual } \\
\text { autorrelatada }\end{array}$ & N (\%) & $\mathrm{AV}^{2}<20 / 40$ \\
\hline & & $\%($ IC95\%) & & $\%$ (IC95\%) \\
\hline Idade & & $\mathrm{p}=0,609^{\star * *}$ & & $\mathrm{p}=0,063^{\star * *}$ \\
\hline 18 a 19 & $763(43,1)$ & $36,7(33,3-40,2)$ & $348(42,9)$ & $5,5(3,5-8,4)$ \\
\hline 20 a 22 & $600(33,9)$ & $36,7(32,9-40,6)$ & $264(32,6)$ & $6,1(3,7-9,7)$ \\
\hline 23 a 39 & $406(23)$ & $39,4(34,8-44,3)$ & $199(24,5)$ & $10,6(7,0-15,7)$ \\
\hline $\operatorname{Sexo}^{*}$ & & $\mathrm{p}<0,001^{\star * *}$ & & $\mathrm{p}=0,907^{\star * *}$ \\
\hline Feminino & $970(54,9)$ & $46,6(43,5-49,8)$ & $440(54,3)$ & $6,8(4,8-9,6)$ \\
\hline Masculino & $797(45,1)$ & $26,0(23,0-29,1)$ & $370(45,7)$ & $7,0(4,8-10,1)$ \\
\hline Estado civil $^{\star *}$ & & $\mathrm{p}=0,386^{* * *}$ & & $\mathrm{p}=0,002$ \\
\hline Solteiro & $1.648(93,1)$ & $37,7(35,4-40,1)$ & $755(93,1)$ & $6,5(4,9-8,5)$ \\
\hline Casado/união estável & $111(6,3)$ & $30,6(22,7-39,9)$ & $52(6,4)$ & $9,6(4,0-21,5)$ \\
\hline Separado/divorciado & $10(0,6)$ & $44,4(14,6-79)$ & $4(0,5)$ & $50,0(4,0-96,0)$ \\
\hline Cor da pele ${ }^{\star}$ & & $\mathrm{p}=0,178^{\star * *}$ & & $\mathrm{p}=0,632^{* * *}$ \\
\hline Branca & $1.271(71,9)$ & $36,3(33,7-39)$ & $595(73,5)$ & $6,7(5,0-9,0)$ \\
\hline Preta & $230(13)$ & $43,9(37,6-50,4)$ & $102(12,6)$ & $5,9(2,6-12,6)$ \\
\hline Parda & $235(13,3)$ & $37,0(31,1-43,4)$ & $101(12,5)$ & $8,9(4,7-16,4)$ \\
\hline Outra $^{3}$ & $31(1,8)$ & $35,5(20,4-54,2)$ & $12(1,5)$ & -- \\
\hline Classe social $^{4^{*}}$ & & $\mathrm{p}=0,043^{\star * *}$ & & $\mathrm{p}=0,416^{\star * *}$ \\
\hline A & $257(15,2)$ & $35,8(30,1-41,9)$ & $111(14,3)$ & $3,6(1,3-9,3)$ \\
\hline B & $740(43,7)$ & $35,0(31,6-38,5)$ & $345(44,3)$ & $8,1(5,7-11,5)$ \\
\hline $\mathrm{C}$ & $620(36,6)$ & $39,7(35,9-43,6)$ & $286(36,7)$ & $6,6(4,3-10,2)$ \\
\hline D-E & $77(4,5)$ & $49,4(38,2-60,5)$ & $37(4,8)$ & $5,4(1,3-20,0)$ \\
\hline Correção óptica & & $\mathrm{p}<0,001^{\star * *}$ & & $\mathrm{p}=0,001^{\star * *}$ \\
\hline Não & $981(55,5)$ & $24,8(22,2-27,6)$ & $489(60,3)$ & $4,5(3,0-6,7)$ \\
\hline Sim & $788(44,5)$ & $52,9(49,4-56,4)$ & $322(39,7)$ & $10,6(7,6-14,4)$ \\
\hline Geral & 1.769 & $37,3(35,1-39,6)$ & 811 & $6,9(5,3-8,9)$ \\
\hline
\end{tabular}

${ }^{1}$ Tabela de Snellen a 6 metros. ${ }^{2} \mathrm{AV}$ : acuidade visual presente. ${ }^{3}$ Categoria inclui cores amarela e indígena. ${ }^{4}$ Classe social determinada de acordo com a Associação Brasileira de Empresas de Pesquisa (ABEP). "Variáveis com missing. Classe social foi a variável com maior percentual de missing $(4,2 \%)$. ${ }^{*}$ Estado Civil: omitida a categoria "viúvo(a)" por ter somente uma observação na amostra geral. ${ }^{* * \star}$ Teste Qui-quadrado.

Fonte: Elaborada pelas autoras.

correção óptica $(55,5 \%)$. A prevalência de dificuldade visual autorrelatada entre eles foi de 37,3\% (IC95\%: 35,1-39,6), sendo maior entre os participantes do sexo feminino (46,6\%; IC95\%: $43,5-49,8)$, os pertencentes às classes sociais D e E (49,4\%; IC95\%: 38,2-60,5) e os usuários de correção óptica(52,9\%; IC95\%: 49,4-56,4).

Participaram da validação 811 indivíduos do consórcio com idade média de 21 anos (DP 3,8). No decorrer do estudo, foi observado que a sensibilidade e a especificidade variavam de acordo com algumas características, portanto, optou-se por estender o estudo de validação, mesmo após atingir o tamanho de amostra calculado previamente $(\mathrm{N}=615)$. As características de tais estudos foram semelhantes às da amostra geral do con- sórcio, exceto para uso de correção óptica (Tabela 2). A prevalência de dificuldade visual autorrelatada nesta amostra foi de 35,8\% (IC95\%: 32,5-39,1). AV menor que 20/40 em qualquer um dos olhos foi encontrada em 6,9\% (IC95\%: 5,3$8,9)$ da amostra do estudo de validação, sendo maior entre indivíduos separados ou divorciados (50,0\%; IC95\%: 4,0-96,0) e entre os usuários de correção óptica (10,6\%; IC95\%: 7,6-14,4) (Tabela 1). No controle de qualidade do teste de acuidade visual obteve-se um kappa igual a 0,87.

A Figura 2 mostra as prevalências de AV alterada em qualquer um dos olhos para cada um dos três critérios utilizados (AV menor que 20/40, menor que 20/70 e menor que 20/200), de acordo com a resposta à pergunta sobre dificuldade 
Tabela 2. Características dos estudantes da Universidade Federal de Pelotas, Rio Grande do Sul, nas amostras dos estudos de prevalência e de validação.

\begin{tabular}{|c|c|c|c|}
\hline Características & $\begin{array}{c}\text { Amostra estudo de } \\
\text { prevalência } \\
\mathrm{N}(\%)\end{array}$ & $\begin{array}{c}\text { Amostra estudo de } \\
\text { validação } \\
\mathrm{N}(\%) \\
\end{array}$ & Valor-p ${ }^{1}$ \\
\hline Idade & & & 0,634 \\
\hline 18 a 19 & $763(43,1)$ & $348(42,9)$ & \\
\hline 20 a 22 & $600(33,9)$ & $264(32,6)$ & \\
\hline 23 a 39 & $406(23)$ & $199(24,5)$ & \\
\hline $\mathrm{Sexo}^{*}$ & & & 0,786 \\
\hline Feminino & $970(54,9)$ & $440(54,3)$ & \\
\hline Masculino & $797(45,1)$ & $370(45,7)$ & \\
\hline Estado civil $^{\star \star}$ & & & 0,966 \\
\hline Solteiro & $1648(93,1)$ & $755(93,1)$ & \\
\hline Casado/união estável & $111(6,3)$ & $52(6,4)$ & \\
\hline Separado/divorciado & $10(0,6)$ & $4(0,5)$ & \\
\hline Cor da Pele ${ }^{*}$ & & & 0,850 \\
\hline Branca & $1271(71,9)$ & $595(73,5)$ & \\
\hline Preta & $230(13)$ & $102(12,6)$ & \\
\hline Parda & $235(13,3)$ & $101(12,5)$ & \\
\hline Outra $^{1}$ & $31(1,8)$ & $12(1,5)$ & \\
\hline Classe social ${ }^{2 *}$ & & & 0,940 \\
\hline A & $257(15,2)$ & $111(14,3)$ & \\
\hline B & $740(43,7)$ & $345(44,3)$ & \\
\hline $\mathrm{C}$ & $620(36,6)$ & $286(36,7)$ & \\
\hline $\mathrm{D}-\mathrm{E}$ & $77(4,5)$ & $37(4,8)$ & \\
\hline Correção óptica & & & 0,021 \\
\hline Não & $981(55,5)$ & $489(60,3)$ & \\
\hline $\operatorname{Sim}$ & $788(44,5)$ & $322(39,7)$ & \\
\hline Total & 1.769 & 811 & \\
\hline
\end{tabular}

visual autorrelatada. Entre aqueles que relataram apresentar dificuldade visual, as prevalências de AV menor que 20/40, menor que 20/70 e menor que 20/200 foram, respectivamente, 13,8\%, 2,8\% e $1,7 \%$. Entre os indivíduos que relataram não apresentar dificuldade visual, estas prevalências foram de $3,1 \%, 0,6 \%$ e $0,4 \%$, respectivamente.

Entre os 56 participantes que apresentavam AV menor que 20/40, 40 afirmaram apresentar dificuldade visual, o que correspondeu a uma sensibilidade de 71,4\% (IC95\%: 57,8-82,7). Já entre aqueles com AV igual ou maior que 20/40, 505 relataram não ter dificuldade visual, o que corresponde a uma especificidade de 66,9\% (IC95\%: 63,4-70,2). Dentre os 290 participantes que relataram apresentar dificuldade visual, 250 foram falsos-positivos, levando a um valor preditivo positivo de 13,8\% (IC95\%: 10,0-18,3) e, dentre os 521 participantes que relataram não ter dificuldade visual, 16 eram falsos-negativos, o que correspondeu a um valor preditivo negativo de 96,9\% (IC95\%: 95,1-98,2). A acurácia da pergunta foi de 67,2\% (IC95\%: 63,9-70,4) (Tabela $3)$. Os valores de sensibilidade, especificidade e valores preditivos positivo e negativo de acordo com as características da amostra estão descritos em material suplementar (Tabela 4), destacando-se a sensibilidade e especificidade por sexo (sensibilidade $83,3 \%$; IC95\% 65,3-94,4 no sexo feminino e $57,7 \%$; IC95\% 36,9-76,6 no sexo masculino; especificidade 57,1\%; IC95\%52,1-61,9 no sexo feminino e 78,5\%; IC $95 \% 73,8-82,7$ no sexo masculino) e entre usuários (sensibilidade 70,6\%; IC95\% 52,5-84,9 e especificidade 50,0\%; IC95\% 44,1-55,9) e não usuários de correção óptica (sensibilidade 72,7\%; IC95\% 49,8-89,3 e especificidade 77,3\%; IC95\% 73,2-81,0). 


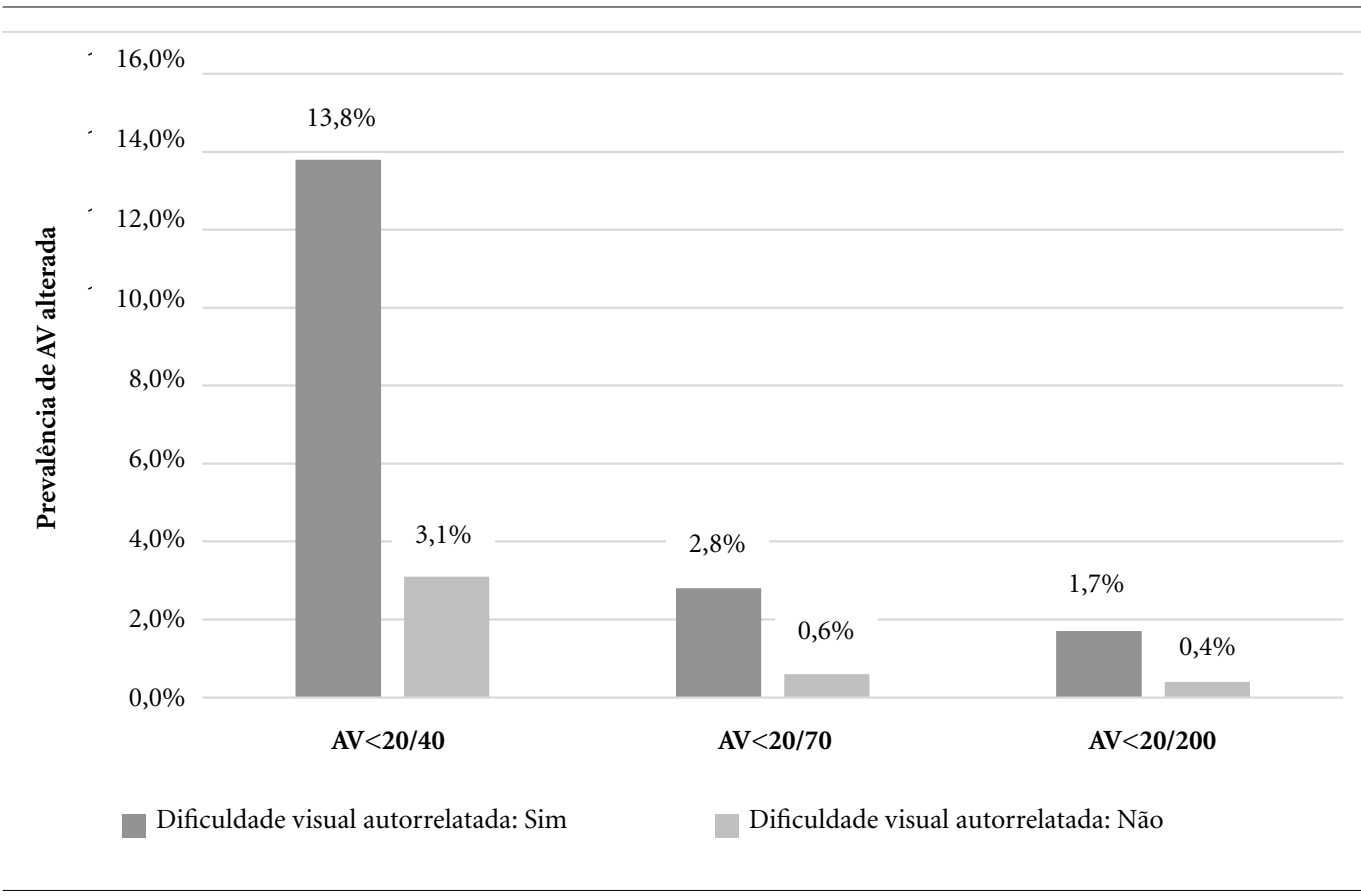

Figura 2. Prevalência de acuidade visual alterada pelo teste padrão-ouro em qualquer um dos olhos para cada um dos três critérios utilizados, de acordo com a resposta à pergunta sobre dificuldade visual autorrelatada.

Fonte: Elaborada pelas autoras.

Tabela 3. Tabela de contingência do estudo de validação e valores de sensibilidade, especificidade, valores preditivos e acurácia para acuidade visual (AV) menor que 20/40 em qualquer olho.

\begin{tabular}{lcc}
\hline & \multicolumn{2}{c}{ AV $<\mathbf{2 0 / 4 0}$} \\
\hline Dificuldade visual autorrelatada & Sim & Não \\
Sim & 40 & 250 \\
Não & 16 & 505 \\
\hline & $\mathbf{\%}$ & IC95\% \\
\hline Sensibilidade & 71,4 & $57,8-82,7$ \\
Especificidade & 66,9 & $63,4-70,2$ \\
VPP & 13,8 & $10,0-18,3$ \\
VPN & 96,9 & $95,1-98,2$ \\
Acurácia & 67,2 & $63,9-70,4$ \\
\hline
\end{tabular}

AV: acuidade visual presente (Tabela de Snellen). VPP: valor preditivo positivo. VPN: valor preditivo negativo.

Fonte: Elaborada pelas autoras.

\section{Discussão}

O presente estudo detectou uma prevalência de $37,3 \%$ de dificuldade visual autorrelatada entre os jovens universitários. Os valores de sensibilidade e especificidade da pergunta estudada fo- ram $71,4 \%$ e $66,9 \%$, e os valores preditivos positivo e negativo $13,8 \%$ e $96,9 \%$, respectivamente.

A prevalência de dificuldade visual autorrelatada foi superior à encontrada em estudo nacional prévio $(5,9 \%)^{4}$. Tal achado pode ser devido à diferença nos termos utilizados (deficiência versus dificuldade), uma vez que deficiência pode ser entendida como um diagnóstico médico prévio, enquanto dificuldade como uma percepção pessoal sobre a visão. Outra hipótese para o achado seria a de que pertencer a uma população universitária possivelmente leva a um maior esforço visual (exigido pelas atividades acadêmicas), o que aumentaria, de alguma forma, a percepção de sua condição visual, ou seja, facilitaria perceber condições oftalmológicas que até então poderiam não ser percebidas. Além disso, a população universitária, por apresentar uma maior escolaridade, pode levar a uma maior percepção de seu estado de saúde.

Estudo realizado no Japão, com 301 indivíduos com idade média de 49 anos submetidos a teste de qualificação para dirigir, mostrou sensibilidade de $73,2 \%$ e especificidade de $35,7 \%$ para uma pergunta única: How well can you see in your daily life?, comparada com $\mathrm{AV}$ menor que $20 / 40^{19}$. Embora a população estudada não seja 
Tabela 4. Sensibilidade, especificidade e valores preditivos positivo e negativo de acordo com as características da amostra do estudo de validação.

\begin{tabular}{|c|c|c|c|c|}
\hline & \multicolumn{4}{|c|}{$\mathrm{AV}^{1}<20 / 40$} \\
\hline & $\begin{array}{l}\text { Sensibilidade } \\
\% \text { (IC95\%) }\end{array}$ & $\begin{array}{c}\text { Especificidade } \\
\%(\mathrm{IC} \%)\end{array}$ & $\begin{array}{c}\mathrm{VPP}^{2} \\
\%(\mathrm{IC} 95 \%)\end{array}$ & $\begin{array}{c}\mathrm{VPN}^{3} \\
\%(\mathrm{IC} 95 \%)\end{array}$ \\
\hline \multicolumn{5}{|l|}{ Idade } \\
\hline 18 a 19 & $57,9(33,5-79,7)$ & $65,7(60,2-70,8)$ & $8,9(4,5-15,3)$ & $96,4(93,1-98,4)$ \\
\hline 20 a 22 & $87,5(61,7-98,4)$ & $69,0(62,8-74,7)$ & $15,4(8,7-24,5)$ & $98,8(95,9-99,9)$ \\
\hline 23 a 39 & $71,4(47,8-88,7)$ & $66,3(58,8-73,2)$ & $20,0(11,6-30,8)$ & $95,2(89,8-98,2)$ \\
\hline \multicolumn{5}{|l|}{ Sexo } \\
\hline Feminino & $83,3(65,3-94,4)$ & $57,1(52,1-61,9)$ & $12,4(8,2-17,8)$ & $97,9(95,2-99,3)$ \\
\hline Masculino & $57,7(36,9-76,6)$ & $78,5(73,8-82,7)$ & $16,9(9,8-26,3)$ & $96,1(93,1-98,0)$ \\
\hline \multicolumn{5}{|l|}{ Cor da pele } \\
\hline Branca & $72,5(56,1-85,4)$ & $68,1(64,1-72,0)$ & $14,1(9,6-19,6)$ & $97,2(95-98,6)$ \\
\hline Preta & $83,3(35,9-99,6)$ & $60,4(49,9-70,3)$ & $11,6(3,9-25,1)$ & $98,3(90,9-100)$ \\
\hline Parda & $66,7(29,9-92,5)$ & $66,3(55,7-75,8)$ & $16,2(6,2-32,0)$ & $95,3(86,9-99,0)$ \\
\hline Outra $^{*}$ & - & - & - & - \\
\hline \multicolumn{5}{|l|}{ Estado civil } \\
\hline Solteiro & $73,5(58,9-85,1)$ & $66,0(62,4-69,5)$ & $13,0(9,3-17,6)$ & $97,3(95,4-98,5)$ \\
\hline Casado/união estável & $60,0(14,7-94,7)$ & $80,9(66,7-90,9)$ & $25,0(5,5-57,2)$ & $95,0(83,1-99,4)$ \\
\hline Separado/divorciado & $50,0(1,3-98,7)$ & $50,0(1,3-98,7)$ & $50,0(1,3-98,7)$ & $50,0(1,3-98,7)$ \\
\hline \multicolumn{5}{|l|}{ Classe social $^{* *}$} \\
\hline A & $75,0(19,4-99,4)$ & $71,0(61,5-79,4)$ & $8,8(1,9-23,7)$ & $98,7(93,0-100)$ \\
\hline B & $57,1(37,2-75,5)$ & $65,3(59,8-70,5)$ & $12,7(7,4-19,8)$ & $94,5(90,6-97,1)$ \\
\hline $\mathrm{C}$ & $89,5(66,9-98,7)$ & $68,5(62,6-74,1)$ & $16,8(10,1-25,6)$ & $98,9(96,1-99,9)$ \\
\hline D-E & $50,0(0,3-98,7)$ & $57,1(39,4-73,7)$ & $6,3(0,2-30,2)$ & $95,2(76,2-99,9)$ \\
\hline \multicolumn{5}{|l|}{ Correção óptica } \\
\hline Não & $72,7(49,8-89,3)$ & $77,3(73,2-81,0)$ & $13,1(7,7-20,4)$ & $98,4(96,5-99,4)$ \\
\hline Sim & $70,6(52,5-84,9)$ & $50,0(44,1-55,9)$ & $14,3(9,4-20,5)$ & $93,5(88,4-96,8)$ \\
\hline Geral & $71,4(57,8-82,7)$ & $66,9(63,4-70,2)$ & $13,8(10,0-18,3)$ & $96,9(95,1-98,2)$ \\
\hline
\end{tabular}

${ }^{1}$ Acuidade visual presente (Tabela de Snellen). ${ }^{2}$ Valor preditivo positivo. ${ }^{3}$ Valor preditivo negativo. ${ }^{\star}$ Categoria inclui cores amarela e indígena. ${ }^{* \star}$ Classe social determinada de acordo com a Associação Brasileira de Empresas de Pesquisa (ABEP).

equivalente entre os estudos, as sensibilidades encontradas em ambos foram superiores a $70 \%$, porém a especificidade foi maior neste presente estudo.

Em estudo norte-americano, Hiller e colaboradores $^{23}$ relataram que $15 \%$ dos indivíduos entre 25 e 74 anos responderam positivamente à questão Do (did) you have trouble with your vision even when wearing glasses or contact lenses?, sendo que esta porcentagem aumentou com a idade. A sensibilidade encontrada variou entre $16 \%$ e $48 \%$, dependendo da idade e severidade da deficiência visual. Já a especificidade foi maior que $80 \%$ em todos os grupos de idade e de severidade da deficiência visual. A sensibilidade foi maior no sexo feminino e não houve diferença entre etnia e nível educacional ${ }^{23}$. Tal achado pode ser consequência de um maior cuidado e melhor percepção de saúde das mulheres em relação aos homens. Em nosso estudo, a sensibilidade não foi maior estatisticamente nos indivíduos de sexo feminino (Tabela 4). Cabe ressaltar, também, a maior prevalência de resposta positiva no presente estudo $(35,8 \%)$.

Houve maior especificidade no sexo masculino (78,5\% vs. $57,1 \%$ no sexo feminino) e entre não usuários de correção óptica $(77,3 \%$ vs. 50,0\% em usuários de correção óptica). A menor proporção de falsos-positivos encontrada nesses grupos pode ser decorrente de uma menor exigência quanto a sua própria visão. A sensibilidade nestes grupos não foi estatisticamente diferente, mas não se pode descartar a possibilidade de falta de poder estatístico para essas comparações.

$\mathrm{O}$ valor preditivo positivo encontrado $(13,8 \%)$, embora baixo, leva a uma probabilidade pós-teste de AV alterada duas vezes maior que a probabilidade pré-teste (prevalência de 6,9\%), 
após uma resposta positiva. Os resultados falsos positivos encontrados podem ser decorrentes da interpretação da pergunta como relativa a outros sintomas oculares, como astenopia, e do ponto de corte utilizado que, embora seja o utilizado pela OMS para deficiência visual, exclui aqueles indivíduos com AV 20/25 a 20/40. O valor preditivo negativo encontrado $(96,9 \%)$ foi alto, porém, não auxilia na definição ou exclusão de casos, uma vez que a probabilidade de não possuir AV alterada passa de $93,1 \%$ pré-teste para $96,9 \%$ após uma resposta negativa à pergunta.

Dentre as limitações deste estudo de validação, é importante destacar que não foi realizado um exame oftalmológico completo para avaliar a saúde ocular geral dos participantes. Além disso, os parâmetros utilizados para cálculo de tamanho de amostra previram uma prevalência de deficiência visual maior do que o encontrado (10\% versus $6,9 \%$ ), levando a uma amostra de tamanho insuficiente para estratificações e com intervalos de confiança de 95\% mais amplos do que se esperava. Outra possível limitação é a definição de AV alterada. Embora os pontos de corte sejam os mesmos utilizados pela OMS, utilizamos a presença de alteração em pelo menos um olho, quando, pelo Código Internacional de Doenças (CID) é a AV no melhor olho que determina a deficiência do indivíduo. A alteração da acuidade visual foi assim definida devido à necessidade de identificação destes indivíduos para adequado encaminhamento a serviço especializado, a fim de corrigir tal alteração. É importante salientar, também, que o estudo foi realizado com universitários e que, portanto, os achados não podem ser extrapolados para indivíduos de 18 a 39 anos que não tenham ingressado na universidade, uma vez que a $\mathrm{AV}$ pode ser influenciada pelos níveis educacionais ${ }^{24}$. É possível que indivíduos

\section{Colaboradores}

JCM Tissot, AMB Menezes e BGC Silva contribuíram na concepção, metodologia e revisão do artigo.

\section{Fonte de financiamento}

O presente trabalho foi realizado com apoio da Coordenação de Aperfeiçoamento de Pessoal de Nível Superior Brasil (CAPES), sob código de financiamento 001 . com menor escolaridade não tenham a mesma percepção de sua AV.

Com relação ao teste de $\mathrm{AV}$, embora o padrão -ouro recomendado para pesquisas clínicas seja a tabela proposta por Bailey-Lovie e, posteriormente, modificada e utilizada a Early Treatment Diabetic Retinopathy Study (ETDRS), para o presente estudo de validação foi utilizada a tabela de Snellen devido à praticidade dessa. Estudo prévio, que comparou a medida da AV com ambas as tabelas, encontrou um coeficiente de determinação de 0,88 entre elas, com uma maior diferença para casos de AV pior do que 20/40025. Assim, a tabela utilizada como padrão-ouro neste estudo não deve ser considerada uma limitação do mesmo.

Entre os pontos fortes do estudo, importante destacar que a pergunta validada e suas opções de respostas são simples e de fácil compreensão. Além disso, importantes requisitos para um estudo de validação foram contemplados ${ }^{26}$ : aplicadora do teste padrão-ouro cega para a resposta e sua realização independente do resultado da pergunta em estudo. Ademais, o controle de qualidade da aplicação do teste padrão-ouro mostrou excelente concordância.

Os resultados deste estudo indicaram uma alta prevalência de dificuldade visual autorrelatada entre jovens universitários. Além disso, a pergunta utilizada mostrou sensibilidade e especificidade razoáveis, podendo ser utilizada para triagem em uma população universitária ou com escolaridade semelhante a do presente estudo que não tem indicação de consulta de rotina com oftalmologista. Ademais, a pergunta em estudo pode ter utilidade, também, para estudos epidemiológicos entre jovens adultos universitários por apresentar sensibilidade e especificidade conhecidas e satisfatórias para este fim.

\section{Referências}

1. Bicas HEA. Acuidade visual: medidas e notaçöes. Arq bras oftalmol 2002; 65: 375-384.

2. Bourne RRA, Flaxman SR, Braithwaite T, Cicinelli MV, Das A, Jonas JB, Keeffe J, Kempen JH, Leasher J, Limburg H, Naidoo K, Pesudovs K, Resnikoff S, Silvester A, Stevens GA, Tahhan N, Wong TY, Taylor HR, Vision Loss Expert Group. Magnitude, temporal trends, and projections of the global prevalence of blindness and distance and near vision impairment: a systematic review and meta-analysis. The Lancet Global health 2017; 5: e888-e97. 
3. Flaxman SR, Bourne RRA, Resnikoff S, Ackland P, Braithwaite T, Cicinelle MV, Das A, Jonas JB, Keeffe J, Kempen JH, Leasher J, Limburg H, Naidoo K, Pesudovs K, Silvester A, Stevens GA, Tahhan N, Wong TY, Taylor HR, Vision Loss Expert Group of the Global Burden od Disease Study. Global causes of blindness and distance vision impairment 1990-2020: a systematic review and meta-analysis. The Lancet 2017; 5: e1221-e34.

4. Malta DC, Stopa SR, Canuto R, Gomes NL, Mendes VLF, Goulart BNG, Moura L Prevalência autorreferida de deficiência no Brasil, segundo a Pesquisa Nacional de Saúde, 2013. Cienc Saude Colet 2016; 21(10): 3253-3264.

5. Abokyi S, Ilechie A, Nsiah P, Darko-Takyi C, Kwasi Abu EK, Osei-Akoto YJ, Youfegan-Baanam M. Visual impairment attributable to uncorrected refractive error and other causes in the Ghanaian youth: The University of Cape Coast Survey. J Optom 2016; 9(1): 64-70.

6. Lemos LEC, Pinheiro Júnior MN. Erros refracionais e sua influência no aprendizado de jovens escolares da Cidade de Manaus. Rev Bras Oftalmol 2002; 61(4): 268-276.

7. Silva CMF, Almeida DR, Bernardes RR, Bazzano FCO, Filho MM, Magalhães CHT, Von Atzingen DANC. Desempenho escolar: interferência da acuidade visual. Rev Bras Oftalm 2013; 72(3): 168-171.

8. Simionato EZR, Rizzon ES, Pires EME, Bassani FR, Ártico LG, Soldera J. Relação da Baixa Acuidade Visual com Reprovação Escolar em crianças do nordeste do Rio Grande do Sul. ACM Arq Catarin Med 2007; 36(3):72-75.

9. Toledo CC, Guerra MR, Leite ICG, Maior MRS, Camilo GB, Paiva APG. Deteç̧ão precoce de deficiência visual e sua relação com o rendimento escolar: study in a early detection of visual impairment and its relation with school effectiveness. Rev Assoc Med Bras (1992) 2010; 56(4): 415-419.

10. Loprinzi PD, Codey K. Influence of visual acuity on anxiety, panic and depression disorders among young and middle age adults in the United States. J Affect Dis 2014; 167: 8-11.

11. Rim TH, Lee CS, Lee SC, Chung B, Kim SS. Influence of visual acuity on suicidal ideation, suicide attempts and depression in South Korea. Br J Ophthalmol 2015; 99(8):1112-1119.

12. Sociedade Brasileira de Oftalmologia (SBO). Importância do Médico Oftalmologista [internet]. [acessado 2017 out 11]. Disponível em: http://sboportal.org.br/ update_materia.aspx?id=5. Rio de Janeiro: SBO; 2017.

13. Canadian Ophthalmological Society evidence-based clinical practice guidelines for the periodic eye examination in adults in Canada. Can J Ophthalmol 2007; 42(1):39-45, 158-163.

14. Feder RS, Olsen TW, Prum Jr. BE, Jr., Summers CG, Olson RJ, Williams RD, Musch DC. Comprehensive adult medical eye evaluation preferred practice pattern guidelines. Ophthalmol 2016; 123(1): 209-236.

15. Revicki D, Rentz A. National Eye Institute Visual Function Questionnaire. Encyclopedia of Quality of Life and Well-Being Research. New York: Springer; 2014.
16. Mangione CM, Phillips RS, Seddon JM, Lawrende MG, Cook EF, Dailey R, Goldman L. Development of the'activities of daily vision scale': a measure of visual functional status. Medical Care 1992; 30(12):11111126.

17. Simão LM, Teixeira AL, Araújo CR, Lana-Peixoto MA, Moreira MA. Versão brasileira do questionário de função visual de 25 itens do questionário do National Eye Institute: tradução, confiabilidade e validação. Arq Bras Oftalmol 2008; 71: 540-546.

18. Duarte WR, Barros AJD, Dias-da-Costa JS, Cattan JM. Prevalência de deficiência visual de perto e fatores associados: um estudo de base populacional. Cad Saude Publica 2003; 19(2): 551-559.

19. Uchino M, Kawashima M, Kaido M, Suwaki K, Uchino Y, Kawachi I, Negishi K, Tsubota K. Evaluation of a paper-based visual acuity questionnaire. Clin Ophthalmol 2017; 11: 1213-1217.

20. Buderer NM. Statistical methodology: I. Incorporating the prevalence of disease into the sample size calculation for sensitivity and specificity. Acad Emerg Med 1996; 3(9): 895-900.

21. Alves ADA. Refração. $6^{\mathrm{a}}$ ed. São Paulo: Grupo Gen Guanabara Koogan; 2000.

22. World Health Organization (WHO). International statistical classification of diseases and related health problems 10th revision. Version: 2016. Geneva: WHO; 2016.

23. Hiller R, Krueger DE. Validity of a survey question as a measure of visual acuity impairment. Am Journal Public Health 1983; 73(1): 93-96.

24. Bicas HEA. Ametropias e presbiopia. Med (Ribeirão Preto) 1997; 30:20-26

25. Kaiser PK. Prospective evaluation of visual acuity assessment: a comparison of snellen versus ETDRS charts in clinical practice (An AOS Thesis). Trans Am Ophthalmol Soc 2009; 107: 311-324.

26. Jaeschke R, Guyatt GH, Sackett DL, Gordon G, Bass E, Brill-Edwards P, Browman G, Cook D, Farkouh M, Gerstein H, Haynes B, Hayward R, Holbrook A, Juniper E, Lee H, Levine M, Moyer V, Nishikawa J, Oxman A, Patel A, Philbrick J, Richardson S, Sauve S, Sackett D, Sinclair J, Trout KS, Tugwell P, Tunis S, Walter S, Wilson M. Users' Guides to the Medical Literature: III. How to Use an Article About a Diagnostic Test B. What Are the Results and Will They Help Me in Caring for My Patients? Jama 1994; 271(9): 703-707.

Artigo apresentado em 29/04/2019

Aprovado em 24/07/2019

Versão final apresentada em 26/07/2019

Editores-chefes: Romeu Gomes, Antônio Augusto Moura da Silva 